\title{
Improved resection and prolonged overall survival with PD-I-IRDye800CW fluorescence probe-guided surgery and PD-I adjuvant immunotherapy in 4TI mouse model
}

\author{
This article was published in the following Dove Press journal: \\ International Journal of Nanomedicine \\ Number of times this article has been viewed
}

\author{
Yang $\mathrm{Du}^{1,2, *}$ \\ Ting Sun ${ }^{3, *}$ \\ Xiaolong Liang ${ }^{4, *}$ \\ Yuan $\mathrm{Li}^{3}$ \\ Zhengyu Jin ${ }^{3}$ \\ Huadan Xue ${ }^{3}$ \\ Yihong Wan ${ }^{5}$ \\ Jie Tian ${ }^{1,2}$ \\ 'CAS Key Laboratory of Molecular \\ Imaging, ${ }^{2}$ The State Key Laboratory \\ of Management and Control for \\ Complex Systems, Institute of \\ Automation, Chinese Academy of \\ Sciences, ${ }^{3}$ Department of Radiology, \\ Peking Union Medical College \\ Hospital, ${ }^{4}$ Department of Ultrasound, \\ Peking University Third Hospital, \\ Beijing, China; ${ }^{5}$ Department of \\ Pharmacology, University of Texas \\ Southwestern Medical Center, \\ Dallas, TX, USA \\ *These authors contributed equally \\ to this work
}

Correspondence: Jie Tian CAS Key Laboratory of Molecular Imaging, Institute of Automation, Chinese Academy of Sciences, No 95 Zhongguancun East Road, Beijing 100190, China

Tel/fax +86 1062527995

Email jie.tian@ia.ac.cn

\section{Yihong Wan}

Department of Pharmacology, University of Texas Southwestern Medical Center, Dallas, TX 75390, USA

Email yihong.wan@utsouthwestern.edu

\begin{abstract}
An intraoperative technique to accurately identify microscopic tumor residuals could decrease the risk of positive surgical margins. Several lines of evidence support the expression and immunotherapeutic effect of PD-1 in breast cancer. Here, we sought to develop a fluorescence-labeled PD-1 probe for in vivo breast tumor imaging and image-guided surgery. The efficacy of PD-1 monoclonal antibody (PD-1 mAb) as adjuvant immunotherapy after surgery was also assessed. PD-1-IRDye $800 \mathrm{CW}$ was developed and examined for its application in tumor imaging and image-guided tumor resection in an immunocompetent 4T1 mouse tumor model. Fluorescence molecular imaging was performed to monitor probe biodistribution and intraoperative imaging. Bioluminescence imaging was performed to monitor tumor growth and evaluate postsurgical tumor residuals, recurrences, and metastases. The PD-1-IRDye $800 \mathrm{CW}$ exhibited a specific signal at the tumor region compared with the IgG control. Furthermore, PD-1-IRDye800CW-guided surgery combined with PD-1 adjuvant immunotherapy inhibited tumor regrowth and microtumor metastases and thus improved survival rate. Our study demonstrates the feasibility of using PD-1-IRDye800CW for breast tumor imaging and image-guided tumor resection. Moreover, PD-1 mAb adjuvant immunotherapy reduces cancer recurrences and metastases emanating from tumor residuals.
\end{abstract}

Keywords: PD-1, programmed cell death-1, near-infrared fluorescence (NIRF), breast cancer, image-guided surgery, immunotherapy, fluorescence imaging

\section{Introduction}

Surgery is the primary treatment modality for most premetastatic solid tumors; however, postsurgical relapse is common and frequently observed as metastases derived from the outgrowth of minimal tumor residuals. Although the role of positive surgical margins in cancer-specific mortality remains controversial, positive margins are a recognized risk factor for tumor recurrence and secondary therapy that may have a great impact on the quality of life. ${ }^{1,2}$ Therefore, imaging techniques that can improve the accurate detection of tumor margins and identification of small cancer foci are urgently needed.

The examination of intraoperative frozen margins is limited by time and prone to sampling error, which can lead to false-negative results. ${ }^{3}$ Thus, an intraoperative technique to accurately identify microscopic tumor residuals could be applied to decrease the risk of positive surgical margins, reduce the rate of re-resection, and tailor adjuvant therapy. ${ }^{4}$ Molecular imaging is an emerging approach to detect microscopic residuals 
within the tumor bed and has been adapted to precisely detect tumors intraoperatively. ${ }^{5}$ Fluorescence optical imaging resolves molecular features, and fluorescence imaging probes can provide contrast between tumor and normal tissues. With tumor-specific probes, breast tumor specimens can be detected, dissected, and then intraoperatively evaluated by image-guided approaches. ${ }^{6}$ The applicability of intraoperative tumor-specific fluorescence imaging for real-time tumor visualization was successfully demonstrated in a first-inhuman analysis in breast cancer. ${ }^{1}$

Studies in mice have demonstrated that residual fluorescence in the tumor margins correlates with local recurrence, whereas image-guided surgery improved outcomes. ${ }^{7}$ Recent clinical studies have supported the use of fluorescence molecular imaging (FMI) during surgery to overcome the challenges associated with precise intraoperative detection of tumor margins. The first clinical application was the use of cetuximab antibody conjugated to the near-infrared fluorescence (NIRF) dye IRDye800CW aimed at showing the feasibility of intraoperative FMI in patients with head and neck squamous cell carcinoma. ${ }^{8}$ More recently, the clinical application of bevacizumab-IRDye800CW combined with intraoperative imaging system improved breast tumor resection. ${ }^{1}$

Although image-guided surgery has shown promise in both preclinical and clinical studies, surgery itself usually results in a generalized state of immunosuppression in the immediate postoperative period that corresponds to tumor progression. ${ }^{9}$ Moreover, resection is often not considered a treatment option for patients with locally advanced tumors or metastases, owing to the high risk of residual outgrowth. Thus, intense attention has been focused on the use of adjuvant therapies such as immunotherapy to eradicate "minimal residual disease" and to prevent postsurgical relapse. ${ }^{10}$

Tumors evade immune surveillance by overexpressing immune checkpoint receptors that negatively regulate T-cell effector function. ${ }^{11,12}$ Immune checkpoint signaling through the PD-1 receptor via its ligand (PD-L1) significantly decreases antitumor immune function. ${ }^{13-15}$ PD-1 is a 288-amino acid, type I transmembrane protein and a member of the B7 receptor immunoglobulin family. PD-1 functions to inhibit antitumor immunity through the expression on activated $\mathrm{T}$ cells, B cells, natural killer cells, and regulatory T cells. ${ }^{16}$ Two ligands have been identified for PD-1/PD-L1 and PD-L2 (also known as B7-H1 and B7-DC, respectively). ${ }^{17,18} \mathrm{PD}-\mathrm{L} 1$ induces T-cell dysfunction upon PD-1 ligation, and its expression is associated with poor outcomes in many solid tumors. ${ }^{19-21}$ Neutralizing antibodies that inhibit PD-1 signaling likely overcome immune resistance, as they have shown unprecedented rates of durable clinical responses in patients with different cancer types. ${ }^{22-24}$ Pretreatment tumor samples obtained from PD-1 blocking therapy showed increased PD-1 and PD-L1 expression within the primary tumor and the invasive margins, which was confirmed by the analysis of expression of the tumor margin marker $\mathrm{S} 100^{+}{ }^{25}$ Therefore, $\mathrm{PD}-1$ was suggested to be an attractive target for antibody-guided fluorescence tumor imaging to identify surgical margins and a potential drug for antitumor immunotherapy.

In the present study, we developed a novel PD-1IRDye $800 \mathrm{CW}$ and explored its application in intraoperative tumor imaging. Furthermore, we also investigated the potential of PD-1 as a target for adjuvant immunotherapy after surgery. To perform these studies, an immunocompetent 4T1 tumor mouse model that recapitulates postresection metastatic relapse was used alongside FMI to monitor in vivo probe biodistribution and intraoperative imaging. Bioluminescence imaging (BLI) was also performed to monitor tumor growth and evaluate tumor residuals, recurrence, and metastasis after surgery.

\section{Materials and methods Synthesis of PD-I-IRDye800CW}

PD-1-IRDye $800 \mathrm{CW}$ was prepared by conjugating IRDye $800 \mathrm{CW}$ succinimidyl ester (LI-COR Biosciences, Lincoln, NE, USA) to PD-1 monoclonal antibody (PD-1 mAb; BioXcell, West Lebanon, NH, USA). Conjugation of IRDye $800 \mathrm{CW}$ to the anti-PD-1 mAb was conducted by using IRDye $800 \mathrm{CW}$ dye from LI-COR Biosciences according to the manufacturer's instructions. Protein concentrations of the IRDye $800 \mathrm{CW}$-conjugated $\mathrm{mAbs}$ and the degree of labeling were determined by measuring absorbance by using an ultraviolet-visible (UV-Vis) spectrophotometer, which was conducted according to the manufacturer's instructions for IRDye ${ }^{\circledR} 800 \mathrm{CW}$ Antibody Labeling from LI-COR Biosciences. Briefly, the PD-1 mAb was first concentrated to $\sim 8.75 \mathrm{mg} / \mathrm{mL}$ using an Amicon 10-kDa Ultra-2 centrifugal filter (Merck Millipore, Billerica, MA, USA). Concentrated anti-PD-1 mAb (10 nmol, $1 \mathrm{~mL}, 0.01 \mathrm{mM})$ was dispersed in borate buffer ( $\mathrm{pH} 8.5-9.0$ ), and aqueous IRDye800CW succinimidyl ester ( $40 \mathrm{nmol}, 0.005 \mathrm{~mL}, 8 \mathrm{mM}$ ) was added to the solution before sequential incubations at room temperature for 2 hours. The resulting solution was purified by centrifugal filtration through $10-\mathrm{kDa}$ filters, and washing with phosphate buffered saline (PBS) buffer ( $\mathrm{pH}$ 7.4) was repeated until no noticeable IRDye $800 \mathrm{CW}$ was detected in the filtrate. The fluorescent probe was then concentrated to $\sim 1 \mathrm{mg} / \mathrm{mL}$ in PBS buffer (pH 7.4) with an Amicon 10-kDa 
centrifugal filter and stored at $-4^{\circ} \mathrm{C}$. IgG2a was also obtained from BioXcell, and IgG2a-IRDye800CW was prepared by using a similar method.

\section{In vitro live cell PD-I-IRDye800CW binding assay}

PD-1-IRDye800CW immunoreactivity was examined in mouse splenic cells and analyzed by fluorescence-activated cell sorting (FACS). Briefly, PD-1-IRDye800CW and unconjugated PD-1 mAb were diluted in FACS buffer at concentrations ranging from $400 \mathrm{ng} / \mathrm{mL}$ to $50 \mu \mathrm{g} / \mathrm{mL}$. Splenic cells were treated with concanavalin $\mathrm{A}(0.5 \mu \mathrm{g} / \mu \mathrm{L}$ for 2 hours $)$ to induce PD-1 expression, and they $\left(1 \times 10^{6}\right)$ were incubated with antibody on ice for 1 hour in 96-well plates. The cells were then washed three times with FACS buffer before staining with secondary antibody for 30 minutes at room temperature. Finally, the cells were analyzed on a flow cytometer (FACSAria ${ }^{\mathrm{TM}}$ III; BD Biosciences, San Jose, CA, USA).

\section{Cell culture and mouse tumor model}

Stable firefly luciferase reporter gene expressing 4T1 (4T1fLuc) mouse mammary tumor cells (American Type Culture Collection, Manassas, VA, USA) were kindly provided by the Radiology Department of Peking Union Hospital. The cells were cultured in Roswell Park Memorial Institute 1640 medium (Hyclone; Thermo Scientific, Rockford, IL, USA) containing $10 \%$ heat-inactivated fetal calf serum (Hyclone; Thermo Scientific) and antibiotics at $37^{\circ} \mathrm{C}$ with $5 \% \mathrm{CO}_{2}$. 4T1-fLuc cells $\left(2 \times 10^{5}\right)$ were injected into the right thigh muscles of 5-week-old female Balb/c mice (18-24 g; Department of Experimental Animals, Peking University Health Science Center, Beijing, China). All experimental protocols were approved by the Institutional Animal Care and Use Committee (IACUC) at Peking University (Permit No: 2011-0039), and all procedures were performed in accordance with the approved guidelines of Peking University IACUC.

\section{Characterization of PD-I expression in tumor tissues}

Western blotting (WB), immunohistochemistry (IHC), and analysis of tumor-infiltrated lymphocyte (TIL) composition can be found in the "Materials and methods" section in the Supplementary materials.

\section{Biodistribution and NIRF imaging with PD-I-IRDye800CW}

FMI was performed to examine the biodistribution and tumor targeting effects of PD-1-IRDye800CW in vivo with the
IVIS Imaging Spectrum System (PerkinElmer Inc., Waltham, MA, USA). Data were dynamically collected from 0 to 48 hours after tail vein injection of PD-1-IRDye $800 \mathrm{CW}$ in 4T1 breast tumor-bearing mice $(n=3)$ and analyzed by using IVIS Living Imaging 3.0 software (PerkinElmer Inc.). IgGIRDye $800 \mathrm{CW}$ was used as control $(\mathrm{n}=3)$. The fluorescence signal was obtained to evaluate the PD-1-IRDye800CW biodistribution and tumor targeting effects. Fluorescence image signals were reported as average radiant efficiency ( $\mathrm{p} / \mathrm{sec} / \mathrm{cm}^{2} / \mathrm{sr}$ ), and tumor-to-background ratio (TBR) was calculated as follows:

$$
\mathrm{TBR}=\frac{\text { Fluorescence light intensity }_{\text {tumor }}}{\text { Fluorescence light intensity }_{\text {muscle }_{\text {e }}}}
$$

\section{Fluorescence image-guided surgery}

Ten 4T1-fLuc tumor-bearing mice were randomly divided into two groups ( $n=5$ in each group) with a tumor volume of $\sim 100 \mathrm{~mm}^{3}$. For the traditional surgery, the surgeons performed surgeries according to their experience and perception without the aid of image guidance. For the FMI-guided surgery group, the mice were intravenously injected with PD-1-IRDye800CW, and real-time intraoperative fluorescence image-guided surgery was performed 24 hours postinjection by a surgeon under a fluorescence stereomicroscope (M205 FA; Leica Microsystems, Wetzlar, Germany), using the NIRF setting (ex 745 nm, em $800 \mathrm{~nm}$ ). The mice were imaged by BLI (IVIS Imaging Spectrum System; Caliper Life Sciences, Waltham, MA, USA) before and after surgery to detect the tumor residuals. Postresection survival was monitored and compared between the PD-1-IRDye800CW and traditional surgery groups over 30-day observation.

\section{PD-I immunotherapy}

The PD-1 mAb therapy, and PBS administration as control, was started immediately after FMI-guided surgery $(200 \mu \mathrm{g}$ intraperitoneal injection, twice per week for 3 weeks). There were five mice in each group, and control mice were administered an equivalent volume of PBS. The tumor recurrences, metastases, and survival were monitored and compared between the PD-1 mAb and PBS treatment groups immediately after FMI-guided surgery over 60-day observation.

\section{Analysis of tumor progression by in vivo $\mathrm{BLI}$}

BLI was performed during drug treatment. Mice were anesthetized with $2 \%$ isoflurane and received an intraperitoneal 
injection of D-luciferin $(80 \mu \mathrm{L} ; 40 \mathrm{mg} / \mathrm{mL}$; Biotium, Fremont, CA, USA) 10 minutes prior to imaging in a lateral position.

\section{Tumor volume and body weight measurement}

The tumor volumes and body weights were measured twice weekly and checked daily, respectively, for clinical signs of illness. Tumor diameters were measured by using a digital caliper and used to calculate tumor volume according to the formula: $1 / 2 \times($ length $) \times(\text { width })^{2}$.

\section{Enzyme-linked immunosorbent assay (ELISA)}

Briefly, blood was collected, incubated at room temperature for 30 minutes, and then centrifuged at 12,000 rpm for 10 minutes. Sera were collected and evaluated for IL-18 and interferon (IFN)- $\gamma$ levels by ELISA (Jiamay Biotech, Beijing, China).

\section{Statistical analyses}

Statistical analyses were performed by using GraphPad Prism software (Version 5.0). Results are presented as the mean \pm standard error of mean from three independent experiments. Two-tailed Student's $t$-tests were performed to compare the means of two groups, and differences between groups were analyzed by using one-way analysis of variance followed by Tukey's multiple comparison test. Overall survival was assessed by using Kaplan-Meier analysis and $\log$ rank test by using Bonferroni correction. $P<0.05$ was considered to indicate statistical significance.

\section{Results \\ PD-I/PD-LI expression in 4TI mouse mammary tumors}

PD-1 and PD-L1 expression in tumors may predict the response to checkpoint blockade therapy. In this study, we characterized PD-1 and PD-L1 protein expression in 4T1 tumors by WB, IHC, and FACS. WB showed that both PD-1 and PD-L1 proteins were expressed in the 4T1 breast tumor homogenates (Figure S1A). The IHC data in Figure S1B suggest that PD-1 and PD-L1 were expressed in the tumor microenvironment (TME; Figure S1Ba and c). IgG staining served as a negative control for PD-1 and PD-L1 (Figure S1Bb and d). To identify the PD-1- and PD-L1-expressing immune cell populations, TILs were analyzed by FACS (Figure S1C). PD-1 was mostly expressed on $\mathrm{CD}^{+} \mathrm{T}$ cells $(27 \%)$, including the $\mathrm{CD}^{+}(27.6 \%)$ and $\mathrm{CD}^{+}(25.6 \%)$ subpopulations, whereas PD-L1 was predominantly expressed on CD11 $\mathrm{b}^{+}$ myeloid-derived suppressor cells $(72 \%)$.

\section{Probe characterization}

The PD-1-IRDye800CW immunoconjugate was prepared by coupling IRDye800CW succinimidyl ester to the lysine groups of PD-1 mAb (Figure 1A). The immunoconjugation reaction resulted in an average of 2.0 IRDye $800 \mathrm{CW}$ molecules per antibody, as determined by UV spectrometry. The ultraviolet-visible-near-infrared (UV-Vis-NIR) absorbance spectrum of PD-1-IRDye800CW in PBS showed a broad absorption at $600-850 \mathrm{~nm}$, with a strong peak at $775 \mathrm{~nm}$, similar to that observed for free IRDye800CW (Figure 1B), indicating the successful conjugation of IRDye800CW to the anti-PD-1 mAb, with no aggregates occurring in the conjugate. Figure $1 \mathrm{C}$ shows the fluorescence emission spectra of aqueous immunoconjugate fluorescent tracers with free IRDye $800 \mathrm{CW}$ used as a control. Notably, both tracers exhibited a fluorescent peak at $\sim 793 \mathrm{~nm}$, which represented a very slight blue shift when compared to free IRDye800CW that peaked at $\sim 795 \mathrm{~nm}$, indicating that the conjugation reaction did not affect IRDye800CW fluorescence. The stability of the conjugates was further investigated. Figure $\mathrm{S} 2$ shows that the PD-1-IRDye800CW and IgG2a-IRDye800CW immunoconjugate were well dispersed in different media including DMEM culture media containing 10\% fetal bovine serum (FBS), PBS, and simulated body fluid. The absorption spectra of PD-1-IRDye800CW in DMEM culture media containing $10 \%$ FBS exhibited almost no change over time up to 1 week, showing high stability of these conjugates (Figure S3).

Moreover, the live-cell-binding affinity of PD-1IRDye $800 \mathrm{CW}$ was examined in mouse splenic cells treated with or without concanavalin A to induce PD-1 expression. Compared with the untreated control cells and isotype IgG antibody (1.1\%-14.7\%; Figure 1Da-d), PD-1-IRDye800CW exhibited higher binding efficiency similar to that observed with unmodified PD-1 expressed on induced splenocytes (50.9\% vs $62.6 \%$; Figure 1 De and $\mathrm{f}$ ). These results suggest that PD-1-IRDye800CW specifically bound PD-1 protein expressed on splenocytes with concanavalin A induction and can be used for in vivo studies.

\section{Dynamic biodistribution of PD-I-IRDye800CW fluorescence probe}

BLI imaging was performed to identify tumor location in mice prior to the injection of fluorescence probe (Figure 2A). FMI results from progressive time points showed that PD-1IRDye $800 \mathrm{CW}$ localized to the tumors by 30 minutes postinjection, gradually accumulated from 2 to 8 hours, and then decreased thereafter with weak signal detected at 48 hours. For the IgG-IRDye800CW control mice, fluorescence signals were also observed with a nonspecific distribution to the 
tumor region 2 to 8 hours after injection, and the tumorlocalized signal decreased until 48 hours. Quantification of TBR for each group showed that the PD-1-IRDye800CW signal was more specific and stable within the tumor region when compared to the IgG control and exhibited its highest TBR at 8 hours, which was $\sim 1.7$-fold higher than the $\operatorname{IgG}$ control at the same time (Figure 2B). To further confirm the in vivo observation, the tumors and major organs were dissected out 48 hours postinjection, and we found that the fluorescence signal of the PD-1-IRDye800CW in the tumors was more specific and stronger than that of the control probe (Figure 2C). Collectively, these results demonstrated that PD-1-IRDye800CW has more specific tumor targeting effects and hence could be used for tumor imaging and intraoperative guidance.

\section{PD-I-IRDye800CW-guided surgery reduced tumor residuals}

Because we found that PD-1-IRDy800CW provided sufficient contrast between tumor and normal tissues, we further investigated its potential application for real-time intraoperative guidance. Image-guided dissection of tumors was performed under a stereo fluorescence microscope 24 hours after an intravenous injection of PD-1-IRDye $800 \mathrm{CW}$ (Figure 3A). NIR imaging was performed before and after resection to assess tumor residuals. As expected, small tumor residuals were found after traditional surgery without intraoperative FMI (Figure 3Ad); however, the use of PD-1-IRDy800CW provided sufficient optical contrast that enabled the complete removal of tumor tissue (Figure 3Ah). BLI was performed to further evaluate the surgical resection and revealed no detectable tumor residuals in the PD-1-IRDy800CW-guided surgery group as indicated by the absence of the BLI signal (Figure 3Bd), whereas some signal remained in the control mice (Figure $3 \mathrm{Bb}$ ). To further investigate the accuracy of the tumor contrast with PD-1-IRDy800CW, histological analyses were performed on resected tissues with tumor border regions between tumor and normal tissues (Figure S4A). The tumor margin shown by PD-1 NIR signal and H\&E staining overlapped at the microscopic level (Figure S4B). These results

A

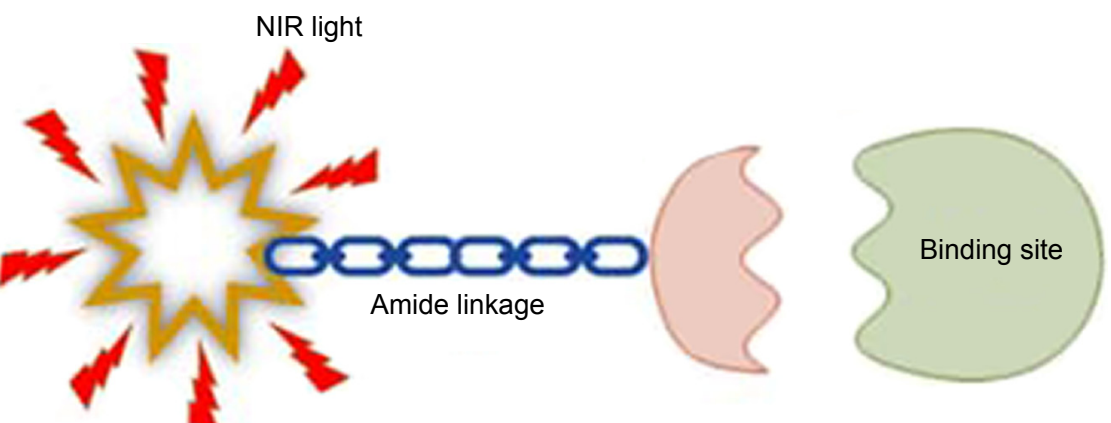

IRDye800CW

PD-1 mAb

B

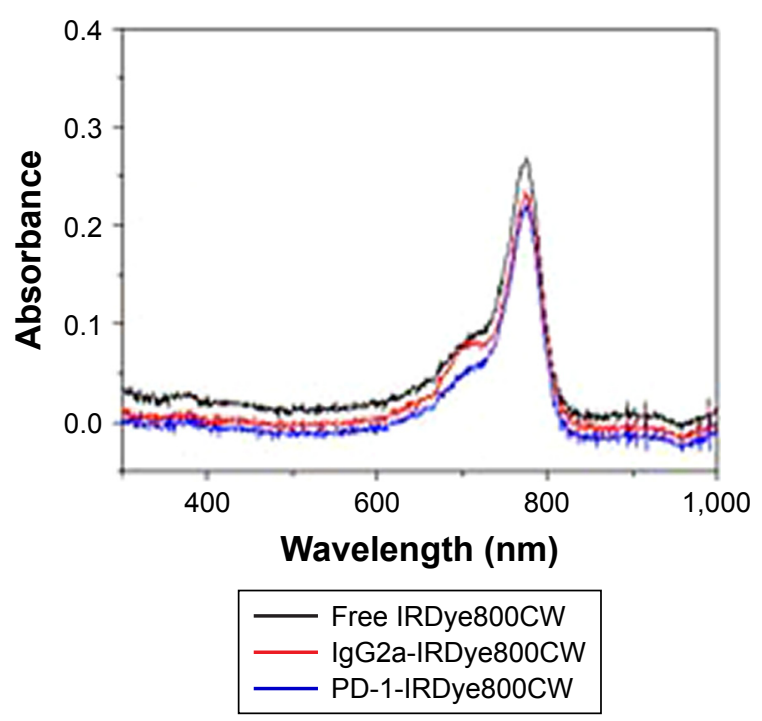

C

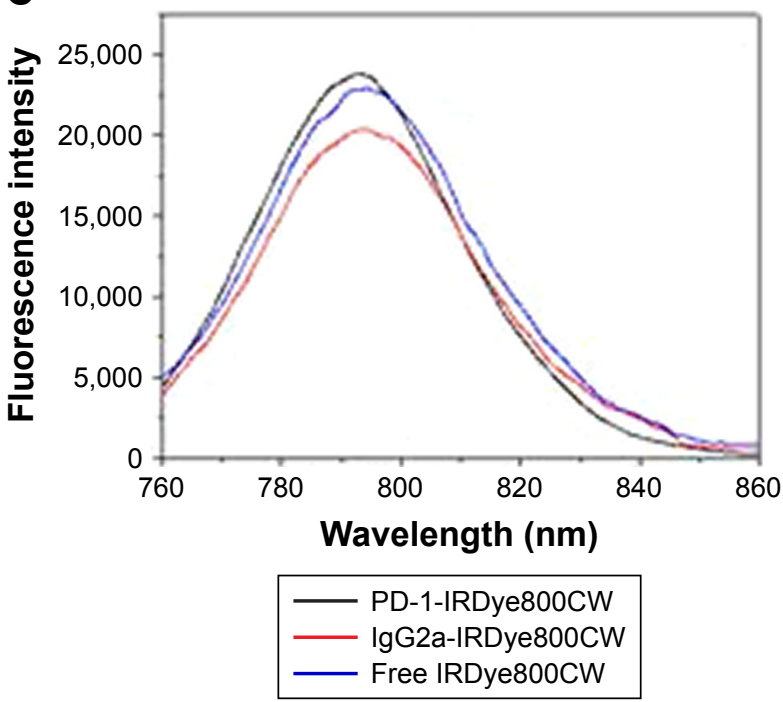

Figure I (Continued) 


\section{D $\quad \lg G$}

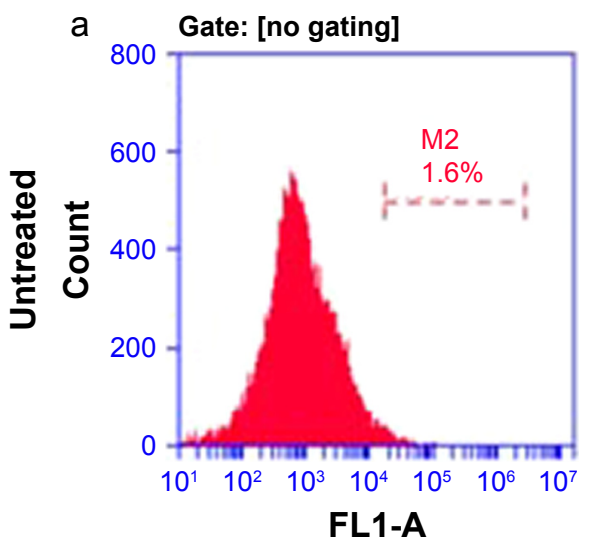

FL1-A

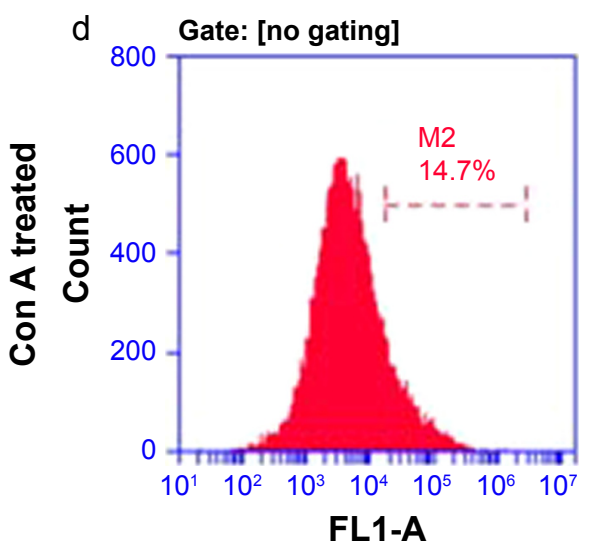

PD-1

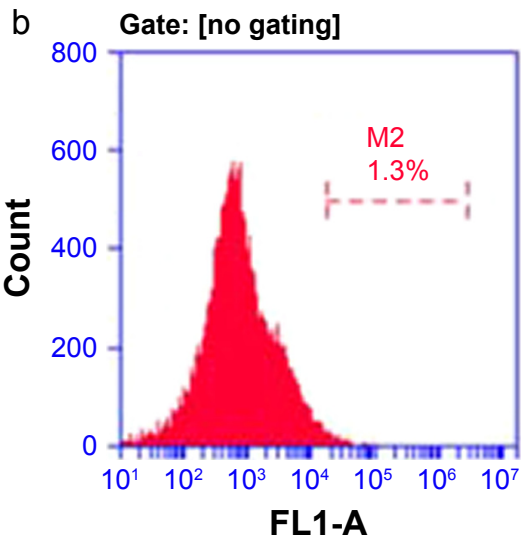

e

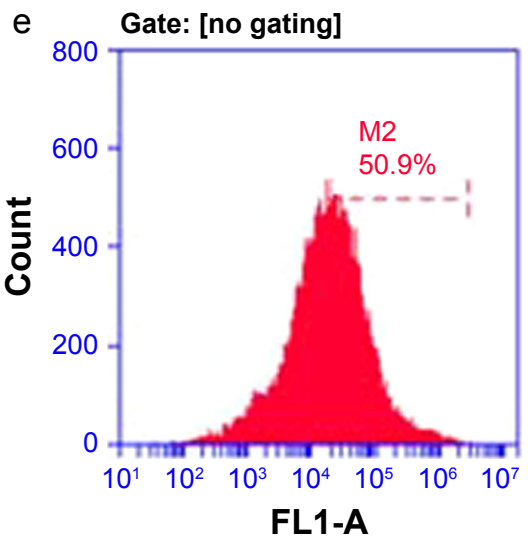

PD-1-IRDye800CW
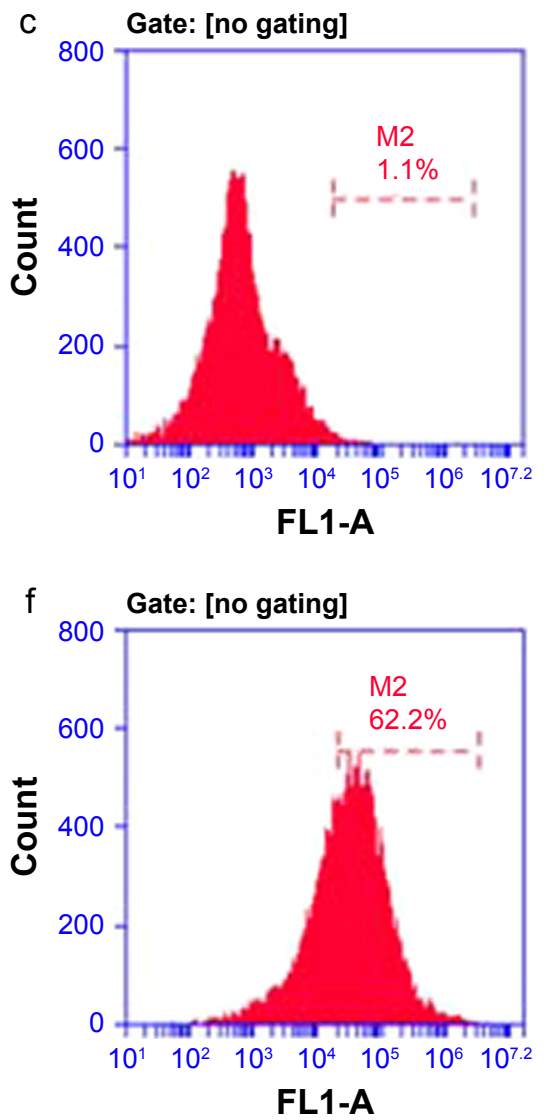

Figure I Characterization of PD-I-IRDye800CW. (A) Schematic representation of the PD-I-IRDye800CW fluorescent tracer. (B) UV-Vis-NIR absorbance spectra of the fluorescent tracers and free dye in PBS. (C) Fluorescence spectra of fluorescent tracers and free dye in water. (D) PD-I-IRDye800CW cell binding was monitored by FACS. $(a-c)$ Untreated splenic cells were stained with isotype control IgG, unmodified PD-I, and PD-I-IRDye800CW. (d-f) Splenic cells treated with Con A were incubated with isotype control IgG, unmodified PD-I, and PD-I-IRDye800CW.

Abbreviations: Con A, concanavalin A; FACS, fluorescence-activated cell sorting; mAb, monoclonal antibody; UV-Vis-NIR, ultraviolet-visible-near-infrared.

suggest that PD-1-IRDy800CW could effectively delineate the tumor margin and improve the precision of tumor resection. Moreover, Kaplan-Meier analysis demonstrated the therapeutic effects of PD-1-labeled fluorescence probe-guided surgery (FMI-guided surgery), as all mice with surgery without FMI guidance succumbed to disease within 30 days, whereas only one FMI-guided surgery-treated mouse died within the same time period (Figure 3C).

\section{Prolonged overall survival with PD-I- IRDye $800 \mathrm{CW}$ fluorescence probe-guided surgery combined with PD-I adjuvant immunotherapy}

In order to assess whether PD-1 adjuvant immunotherapy could further enhance antitumor effects after PD-1-IRDy800CW FMI-guided surgery, mice were divided into PD-1 adjuvant immunotherapy and PBS control groups $(n=5)$ immediately after surgery (Figure 4A). Notably, PD-1 treatment did not induce any loss in body weight, suggesting that the dosing regimen was relatively safe and well tolerated (Figure 4B). Survival analysis demonstrated that PD-1 mAb treatment prolonged overall survival as all control mice succumbed to disease within 40 days, whereas those administered with PD-1 mAb survived for $>50$ days (Figure 4C). Moreover, as 4T1 mammary tumors are known to be generally prone to metastasis, we found that four of five control mice developed tumor metastases to the lung and spinal cord, whereas only one PD-1 mAb-treated mouse exhibited lung metastasis as determined by BLI (Figure 4D). Thus, these data support that PD-1-IRDye800CW FMI-guided surgery combined with PD-1 adjuvant immunotherapy reduced the incidence of microtumor metastases and prolonged overall survival with no obvious side effects.

\section{PD-I immunotherapeutic effects occur} via IFN- $\gamma$ and IL- I 8 cytokine upregulation IFN- $\gamma$ and IL-18 cytokine secretion was monitored by ELISA to gain a better understanding of the immune-mediated response 
A BLI

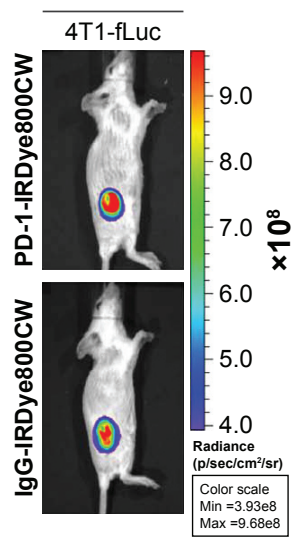

FMI

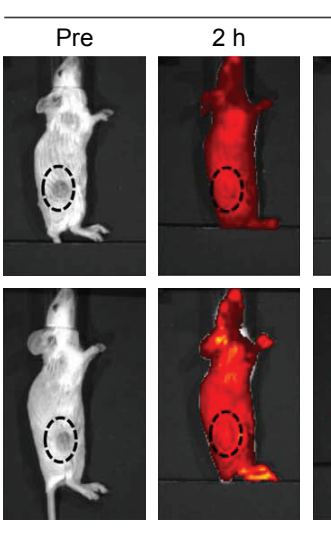

$8 \mathrm{~h}$
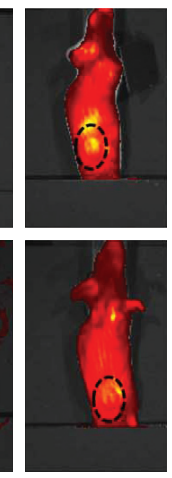
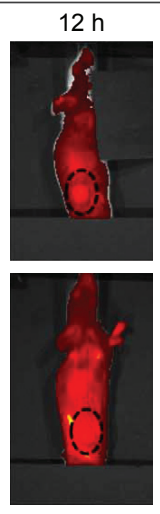

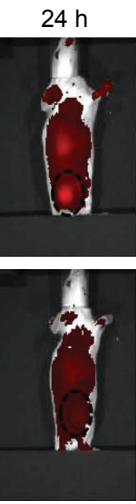

B

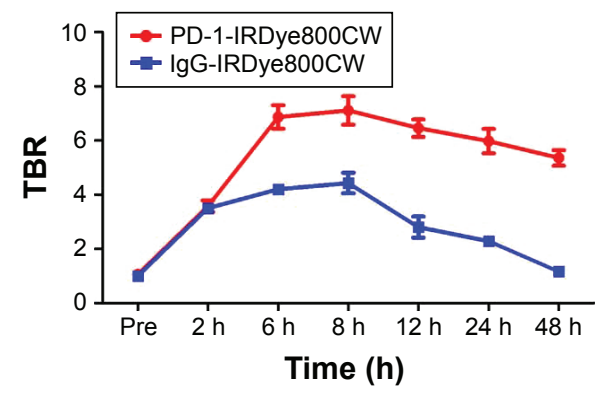

C

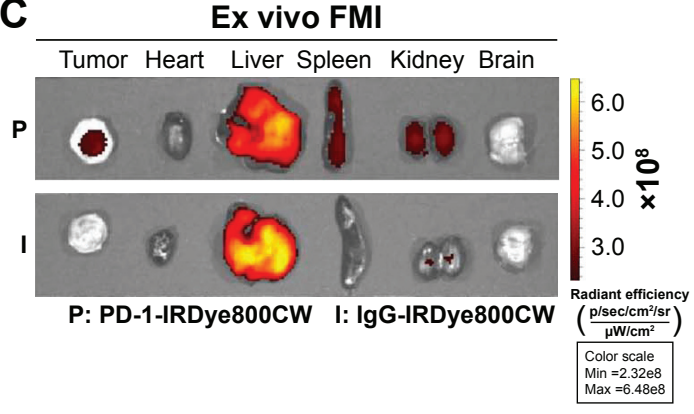

Figure 2 Analysis of PD-I-IRDye800CW biodistribution in 4TI-fLuc mammary tumor-bearing mice by FMI. (A) FMI of tumor-bearing mice injected with PD-I-IRDye800CW or IgG-IRDye $800 \mathrm{CW}(\mathrm{n}=3)$. BLI was used to denote the location of tumors. (B) Quantification of in vivo FMI TBR. (C) Ex vivo FMI of tumors and internal organs 8 hours after probe injection. The dotted circle indicates tumor location.

Abbreviations: 4TI-fLuc, firefly luciferase reporter gene expressing 4TI; BLI, bioluminescence imaging; FMI, fluorescence molecular imaging; TBR, tumor-to-background ratio.

to PD-1 treatment. The serum IFN- $\gamma$ level was $\sim 3$-fold higher in PD-1 mAb-treated mice than in the PBS control mice. Because IFN- $\gamma$ stimulates IL-18 expression, it was not surprising that IL-18 levels were $\sim 1.5$-fold higher in PD-1 mAbtreated mice than in the PBS control mice (Figure 5). Thus, these results indicate that a systemic host immune response to PD-1 pathway inhibition may drive the antitumor effects.

\section{Discussion}

This study provides proof of principle for image-guided surgical resection with PD-1-IRDye800CW in breast cancer. In addition, enhanced antitumor effects were observed when adjuvant PD-1 immunotherapy was administered after resection. To the best of our knowledge, this is the first time that an NIRF-labeled PD-1 antibody has been used as a targeted probe for image-guided surgery, and PD-1 has been used as adjunctive immunotherapy after surgery.

Currently, there is an unmet need for a real-time method to identify tumor boundaries during the initial resection, which can reduce the need for subsequent surgery and reduce the risk of local recurrence. FMI is a particularly attractive approach to detect tumor residuals, as it can provide cellular resolution with several millimeters of penetration. ${ }^{6,26} \mathrm{PD}-1$ is an immune inhibitory receptor expressed by activated T cells, which play a pivotal role in tumor immune evasion. Importantly, previous work demonstrated that samples obtained from patients responding to PD-1 treatment showed higher numbers of PD-1-, PD-L1-, and CD8-expressing cells at the invasive tumor margin and also inside tumors, with a close proximity between PD-1 and PD-L1. ${ }^{25}$ This work supported the use of PD-1 as a potential biomarker to delineate the tumor periphery from the surrounding normal tissue. Therefore, in the present study, we developed a PD-1-targeted fluorescence probe for intraoperative imaging during tumor resection. FMI analysis showed that PD-1-IRDye800CW exhibited more specific targeting and longer retention in the tumor tissue when compared to the IgG isotype control. Most previous studies mainly utilized nuclear-labeled PD-1 mAb for in vivo tumor imaging. ${ }^{27}$ Our PD-1-IRDye $800 \mathrm{CW}$ NIRF probe is new and safer than nuclear imaging and possesses the potential for use in intraoperative FMI-guided surgery. Thus, the image-guided surgery was performed utilizing the PD-1-IRDye800CW fluorescence probe in this study, and the post-resection BLI data revealed that intraoperative guidance with the PD-1 fluorescence probe enabled a more precise resection with the absence of detectable tumor residuals. ${ }^{27}$ 
A

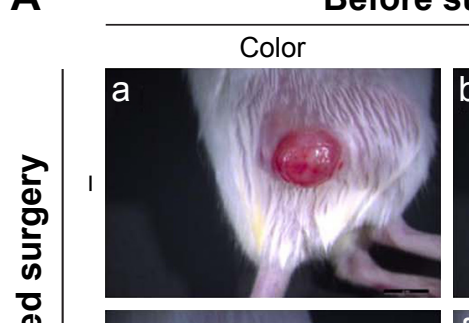

Before surgery
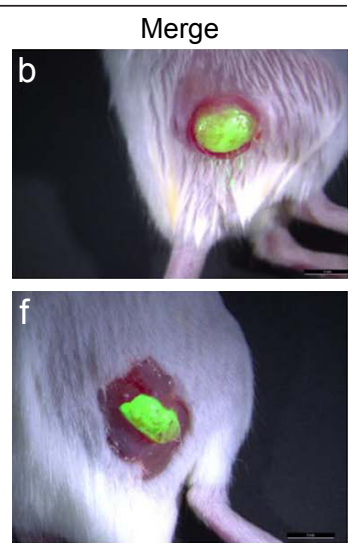

After

B
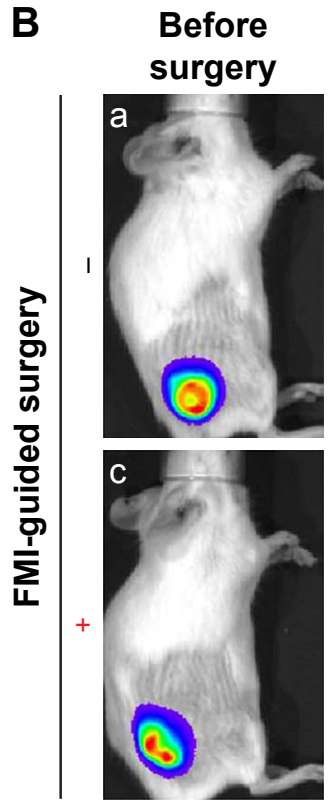
surgery

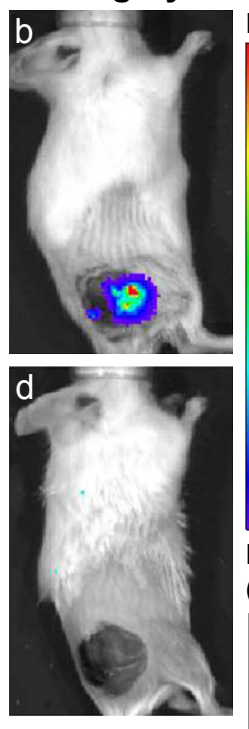

Luminescence

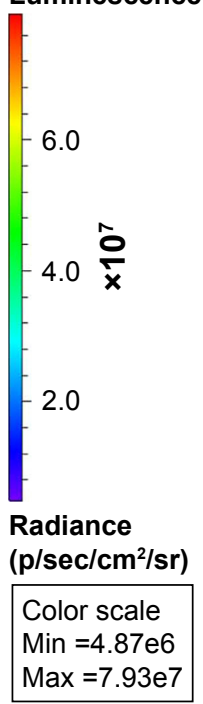

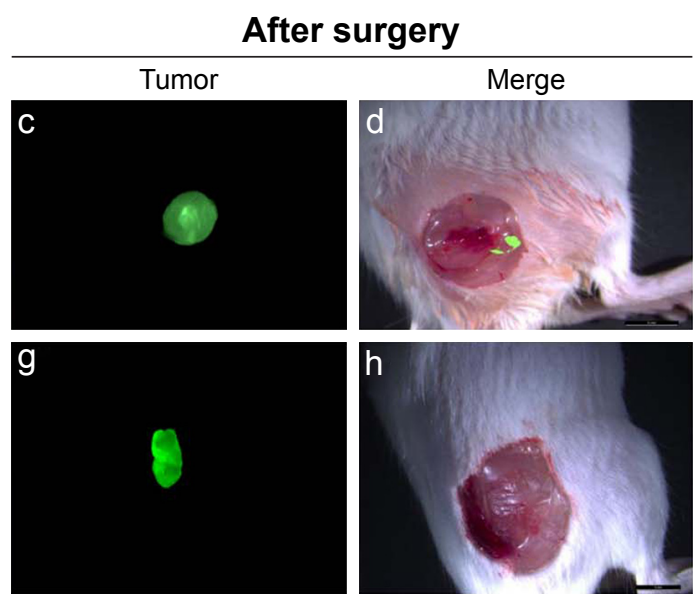

C

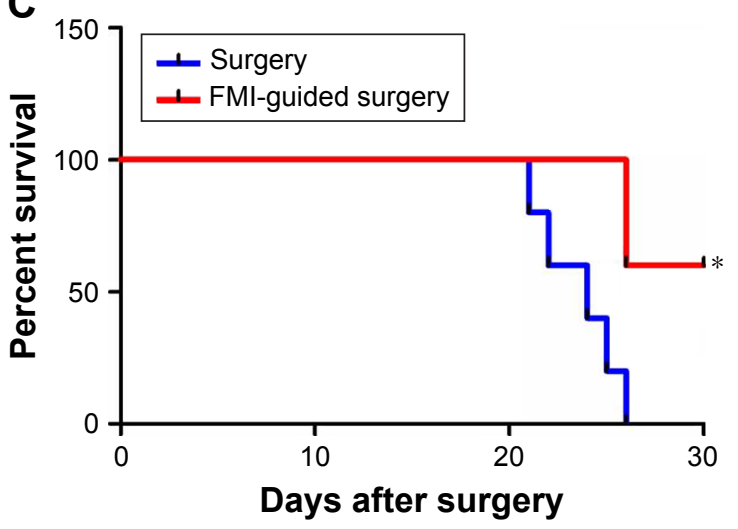

Figure $3 \mathrm{FMI}$-guided surgery with PD-I-IRDye800CW and evaluation of tumor residuals $(\mathrm{n}=4)$. (A) White light and fluorescence images before and after tumor resection without $(\mathrm{a}-\mathrm{d})$ or with $(\mathrm{e}-\mathrm{h})$ FMI-guided surgery. (B) Tumor residuals were further evaluated by BLI before $(\mathrm{a}, \mathrm{c})$ and after surgery $(\mathrm{b}, \mathrm{d})$. Scale bar $=50 \mu \mathrm{m}$. $(\mathbf{C})$ Comparison of survival rate with or without FMl-guided surgery. $* P<0.05$.

Abbreviations: BLI, bioluminescence imaging; FMI, fluorescence molecular imaging.

For certain cancers, including breast cancer, administration of adjuvant therapies immediately after resection has led to marked improvements in treatment outcomes. Some breast cancers, particularly the triple-negative subtype, are generally aggressive tumors with a high rate of distant metastasis and poor overall survival when identified at a later stage. The limited effectiveness of surgical resection and conventional therapies has encouraged the development of immunotherapies that might be useful as adjuvants. To date, preclinical studies have shown that adjuvant immunotherapy with surgery, chemotherapy, and/or radiotherapy is generally the most effective approach to treat cancer. ${ }^{28}$ For instance, PD-1 blockade can mediate tumor regression in patients with various cancers by inhibiting adaptive immune resistance. Expression analysis revealed that PD-L1 was present in $34 \%$ of breast tumors and associated with high-risk clinicopathological features. ${ }^{29}$ Moreover, in triple-negative breast cancer patients, there were a significantly higher number of intratumoral $\mathrm{CD} 8^{+} \mathrm{T}$ cells in tumors that were PD-L1 ${ }^{+}$compared with that in PD-L1 $1^{-}$tumors, suggesting a strong association of PD-1/ PD-L1 signaling with breast tumor. ${ }^{30}$ In this preclinical study, we found that post-resection PD-1 adjuvant immunotherapy significantly reduced breast tumor recurrence and metastasis, resulting in prolonged overall survival. It is worth noting that the prognosis may not be different between groups that undergo immunotherapy after complete resection with or without FMI guidance, and the current concept cannot be applied in the case of tumors not related to PD-1/PD-L1 signals. 
A
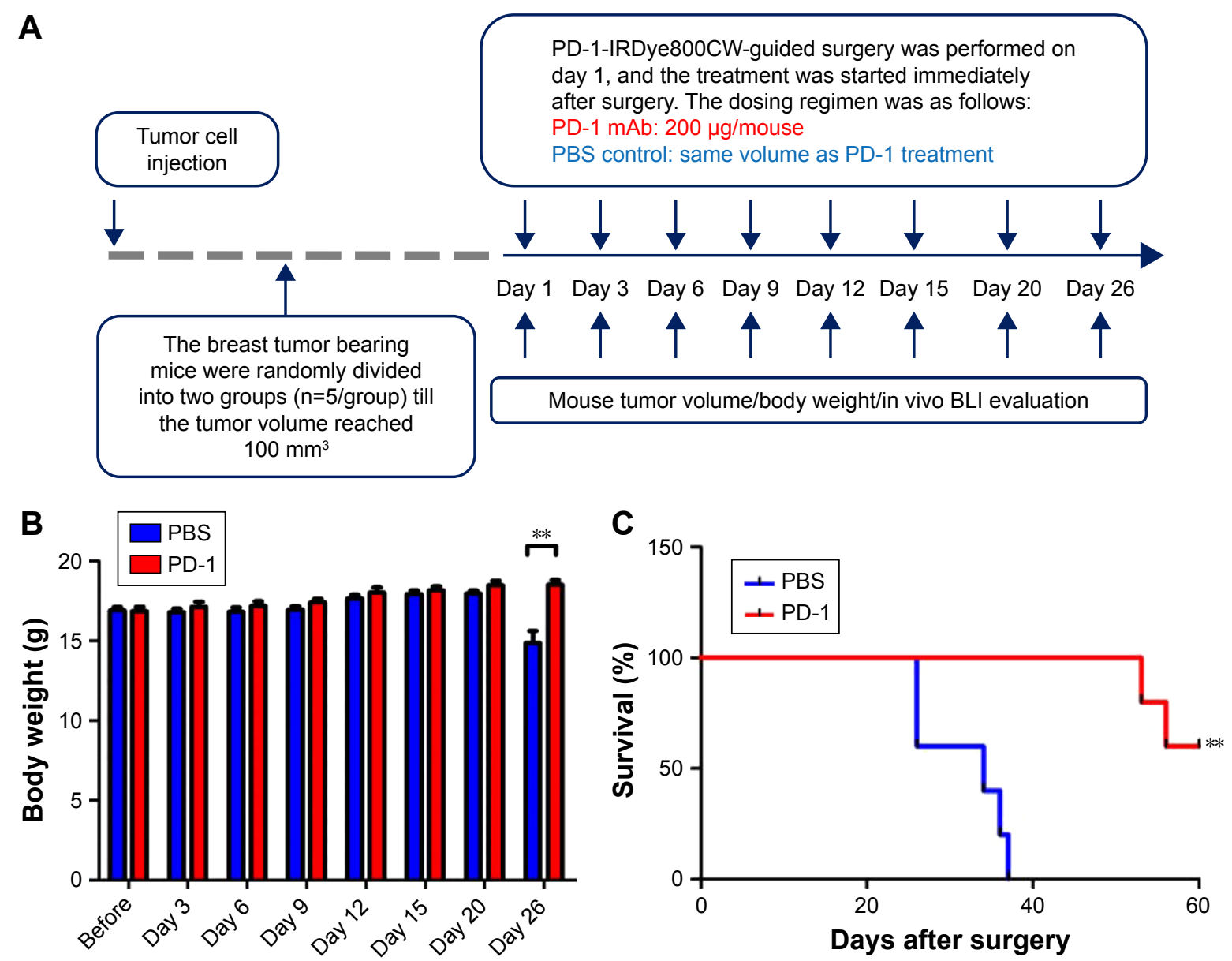

\section{Days after surgery}

D

\begin{tabular}{|c|c|c|c|c|c|c|c|}
\hline Mice & Metastasis & Day 1 & Day 3 & Day 6 & Day 9 & Day 12 & $\begin{array}{l}\text { Survival time } \\
\text { after surgery }\end{array}$ \\
\hline PBS-1 & $\sqrt{ }$ & & & & & Lung & 26 \\
\hline PBS-2 & $\sqrt{ }$ & & & Lung & & & 26 \\
\hline PBS-3 & $\sqrt{ }$ & & & & Lung, spinal cord & & 34 \\
\hline PBS-4 & $\sqrt{ }$ & & & & & Spinal cord & 36 \\
\hline PBS-5 & $x$ & & & & & & 37 \\
\hline PD-1-1 & $x$ & & & & & & 53 \\
\hline PD-1-2 & $x$ & & & & & & 56 \\
\hline PD-1-3 & $x$ & & & & & & $>60$ \\
\hline PD-1-4 & $\sqrt{ }$ & & & Lung & & & $>60$ \\
\hline PD-1-5 & $x$ & & & & & & $>60$ \\
\hline
\end{tabular}

Figure 4 PD-I immunotherapy combined with PD-I image-guided surgery inhibited tumor relapse and increased overall survival. (A) Experimental schematic representation of the 4TI tumor model. Body weight (B), survival (C), and metastasis (D) were monitored after surgery. $* * P<0.01$.

Abbreviations: $\mathrm{BLI}$, bioluminescence imaging; $\mathrm{mAb}$, monoclonal antibody; PBS, phosphate buffered saline.

The potential mechanism for the antitumor effects observed with PD-1 treatment was also investigated in this study. We found significantly higher serum IL-18 and IFN- $\gamma$ levels in PD-1 mAb-treated mice than in the control group, suggesting that an immune response was elicited after PD-1 blockade. ${ }^{31}$ Previous reports demonstrated that PD-1 mAb treatment elicits and functions in the proliferation of naive and memory $\mathrm{CD} 8^{+} \mathrm{T}$ cells after PD- $1 \mathrm{mAb}$ treatment. ${ }^{32}$ Thus, the results are indicative of a potential systemic host response to PD-1 pathway inhibition. 
A

IFN- $\gamma$

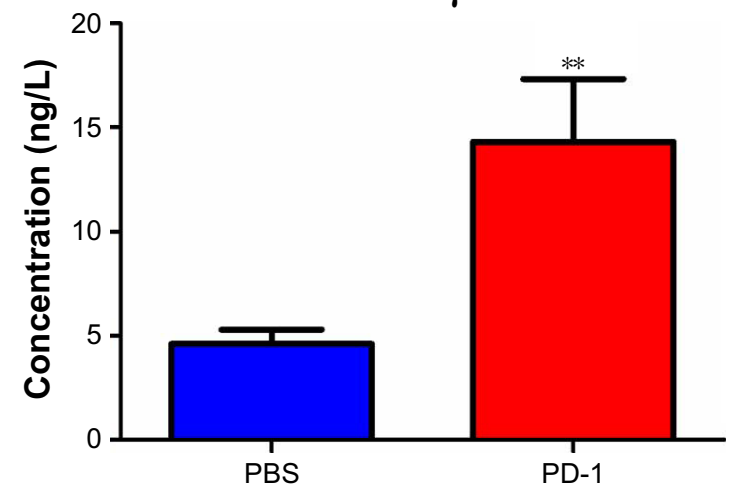

B

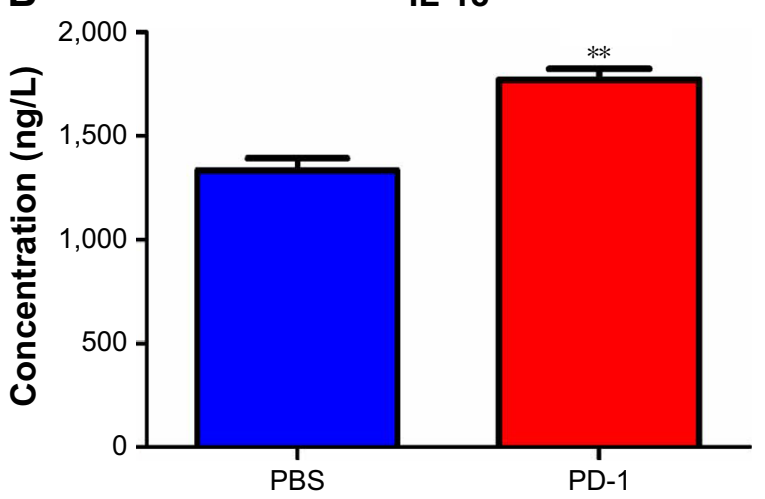

Figure 5 Serum IFN- $\gamma(\mathbf{A})$ and IL-18 (B) levels of PD-I mAb-treated mice and PBS controls were monitored by ELISA. $* * P<0.0$ I. Abbreviations: ELISA, enzyme-linked immunosorbent assay; IFN- $\gamma$, interferon- $\gamma$; mAb, monoclonal antibody; PBS, phosphate buffered saline.

In addition, anti-PD-1 mAb treatment shows an acceptable toxicity profile in patients, including those with nonsmall-cell lung cancer, melanoma, and renal cell cancer., ${ }^{5,12}$ Here, PD-1 mAb-treated mice were devoid of body weight loss and signs of morbidities, consistent with previous reports. Moreover, treated mice displayed significantly prolonged survival compared with control mice, suggesting that the dosage and regimen of PD-1 mAb administration were tolerable and effective as an immunotherapy.

\section{Conclusion}

We report that concurrent PD-1-IRDye800CW-guided surgery and PD-1 adjuvant immunotherapy produce potent and durable antitumor effects in a 4T1 mammary mouse model. The PD-1 is mainly expressed in the TILs in the TME; therefore, our imaging and therapeutic strategy possesses the capacity to image the expression of PD- 1 in TME in a variety of tumors in immunocompetent models. The development of a noninvasive PD-1 imaging approach for the quantitative measurement of PD-1 expression is urgently needed, given the recent clinical development and approval of PD-1 therapeutic antibodies, unresolved problems with traditional biopsy/IHC approaches, the impracticality of repetitive tissue sampling for the advanced disease stage, and dynamic changes of PD-L1 expression in the TME. Furthermore, the noninvasive FMI imaging approach is relatively safer than nuclear imaging and could enhance immune checkpoint therapy management and reduce immune-related toxicities. Further investigation is needed to evaluate the potential of PD-1 as both a cancer immunodiagnostic and a therapeutic agent.

\section{Acknowledgments}

This work was supported by the National Natural Science Foundation of China (81227901, 81470083, 81527805, $81571810,61231004,81771846$, and 81201186), the Research and Development Program of China (973) under Grants 2014CB748600 and 2015CB755500, the Strategic Priority Research Program from Chinese Academy of Sciences (XDB02060010), Beijing Natural Science Foundation (Z16110200010000), a grant from Peking University Third Hospital (BYSY2015023), the China Postdoctoral Science Foundation (2013M530014), Cancer Prevention Research Institute of Texas (RP130145, YW), US Department of Defense (W81XWH-13-1-0318, YW), Welch Foundation (I-1751, YW), and Mary Kay Foundation (\#073.14, YW). The abstract of this paper was presented at the American Association for Cancer Research Conference, named "Improved resection and prolonged overall survival with PD-1IRDye800CW fluorescent probe-guided surgery and PD-1 adjuvant immunotherapy" as a poster presentation with interim findings. The poster's abstract was published in "Poster Abstracts" in Cancer Research Journal (Published first July 2, 2017, Volume 77, Issue 13 Supplement, pp. 875; doi: 10.1158/1538-7445.AM2017-875).

\section{Disclosure}

The authors report no conflicts of interest in this work.

\section{References}

1. Lamberts LE, Koch M, de Jong JS, et al. Tumor-specific uptake of fluorescent bevacizumab-IRDye $800 \mathrm{CW}$ microdosing in patients with primary breast cancer: a phase I feasibility study. Clin Cancer Res. 2017; 23(11):2730-2741.

2. Unkart JT, Chen SL, Wapnir IL, Gonzalez JE, Harootunian A, Wallace AM. Intraoperative tumor detection using a ratiometric activatable fluorescent peptide: a first-in-human phase 1 study. Ann Surg Oncol. Epub 2017 Jul 11.

3. Cendan JC, Coco D, Copeland EM 3rd. Accuracy of intraoperative frozen-section analysis of breast cancer lumpectomy-bed margins. J Am Coll Surg. 2005;201(2):194-198.

4. Nguyen QT, Olson ES, Aguilera TA, et al. Surgery with molecular fluorescence imaging using activatable cell-penetrating peptides decreases residual cancer and improves survival. Proc Natl Acad Sci U S A. 2010; 107(9):4317-4322. 
5. Weissleder R, Pittet MJ. Imaging in the era of molecular oncology. Nature. 2008;452(7187):580-589.

6. Whitley MJ, Cardona DM, Lazarides AL, et al. A mouse-human phase 1 co-clinical trial of a protease-activated fluorescent probe for imaging cancer. Sci Transl Med. 2016;8(320):320ra4.

7. Chi C, Zhang Q, Mao Y, et al. Increased precision of orthotopic and metastatic breast cancer surgery guided by matrix metalloproteinaseactivatable near-infrared fluorescence probes. Sci Rep. 2015;5:14197.

8. Rosenthal EL, Warram JM, de Boer E, et al. Safety and tumor specificity of cetuximab-IRDye 800 for surgical navigation in head and neck cancer. Clin Cancer Res. 2015;21(16):3658-3666.

9. Hogan BV, Peter MB, Shenoy HG, Horgan K, Hughes TA. Surgery induced immunosuppression. Surgeon. 2011;9(1):38-43.

10. Kwon ED, Foster BA, Hurwitz AA, et al. Elimination of residual metastatic prostate cancer after surgery and adjunctive cytotoxic $\mathrm{T}$ lymphocyte-associated antigen 4 (CTLA-4) blockade immunotherapy. Proc Natl Acad Sci U S A. 1999;96(26):15074-15079.

11. Drake CG, Jaffee E, Pardoll DM. Mechanisms of immune evasion by tumors. Adv Immunol. 2006;90:51-81.

12. Fridman WH, Pages F, Sautes-Fridman C, Galon J. The immune contexture in human tumours: impact on clinical outcome. Nat Rev Cancer. 2012;12(4):298-306.

13. Nguyen LT, Ohashi PS. Clinical blockade of PD1 and LAG3-potential mechanisms of action. Nat Rev Immunol. 2015;15(1):45-56.

14. Ohaegbulam KC, Assal A, Lazar-Molnar E, Yao Y, Zang X. Human cancer immunotherapy with antibodies to the PD-1 and PD-L1 pathway. Trends Mol Med. 2015;21(1):24-33.

15. Topalian SL, Hodi FS, Brahmer JR, et al. Safety, activity, and immune correlates of anti-PD-1 antibody in cancer. NEngl J Med. 2012;366(26): 2443-2454.

16. Agata Y, Kawasaki A, Nishimura H, et al. Expression of the PD-1 antigen on the surface of stimulated mouse $\mathrm{T}$ and B lymphocytes. Int Immunol. 1996;8(5):765-772.

17. Butte MJ, Keir ME, Phamduy TB, Sharpe AH, Freeman GJ. Programmed death-1 ligand 1 interacts specifically with the B7-1 costimulatory molecule to inhibit T cell responses. Immunity. 2007;27(1):111-122.

18. Flies DB, Sandler BJ, Sznol M, Chen L. Blockade of the B7-H1/PD-1 pathway for cancer immunotherapy. Yale J Biol Med. 2011;84(4): 409-421.

19. Topalian SL, Sznol M, McDermott DF, et al. Survival, durable tumor remission, and long-term safety in patients with advanced melanoma receiving nivolumab. J Clin Oncol. 2014;32(10):1020-1030.
20. Wolchok JD, Kluger H, Callahan MK, et al. Nivolumab plus ipilimumab in advanced melanoma. $N$ Engl J Med. 2013;369(2):122-133.

21. Brahmer JR, Tykodi SS, Chow LQ, et al. Safety and activity of antiPD-L1 antibody in patients with advanced cancer. N Engl J Med. 2012; 366(26):2455-2465.

22. Hamid O, Robert C, Daud A, et al. Safety and tumor responses with lambrolizumab (anti-PD-1) in melanoma. $N$ Engl J Med. 2013; 369(2):134-144.

23. Okazaki T, Chikuma S, Iwai Y, Fagarasan S, Honjo T. A rheostat for immune responses: the unique properties of PD-1 and their advantages for clinical application. Nat Immunol. 2013;14(12):1212-1218.

24. Radvanyi L, Pilon-Thomas S, Peng W, et al. Antagonist antibodies to PD-1 and B7-H1 (PD-L1) in the treatment of advanced human cancerletter. Clin Cancer Res. 2013;19(19):5541.

25. Tumeh PC, Harview CL, Yearley JH, et al. PD-1 blockade induces responses by inhibiting adaptive immune resistance. Nature. 2014 515(7528):568-571.

26. Sheth RA, Arellano RS, Uppot RN, et al. Prospective trial with optical molecular imaging for percutaneous interventions in focal hepatic lesions. Radiology. 2015;274(3):917-926.

27. Natarajan A, Mayer AT, Xu L, Reeves RE, Gano J, Gambhir SS Novel radiotracer for immunoPET imaging of PD-1 checkpoint expression on tumor infiltrating lymphocytes. Bioconjug Chem. 2015; 26(10):2062-2069.

28. Mellman I, Coukos G, Dranoff G. Cancer immunotherapy comes of age. Nature. 2011;480(7378):480-489.

29. Mittendorf EA, Philips AV, Meric-Bernstam F, et al. PD-L1 expression in triple-negative breast cancer. Cancer Immunol Res. 2014; 2(4):361-370.

30. Chawla A, Philips AV, Alatrash G, Mittendorf E. Immune checkpoints: a therapeutic target in triple negative breast cancer. Oncoimmunology. 2014;3(3): 28325

31. Herbst RS, Soria JC, Kowanetz M, et al. Predictive correlates of response to the anti-PD-L1 antibody MPDL3280A in cancer patients. Nature. 2014;515(7528):563-567.

32. Barber DL, Wherry EJ, Masopust D, et al. Restoring function in exhausted CD8 T cells during chronic viral infection. Nature. 2006; 439(7077):682-687. 


\section{Supplementary materials \\ Materials and methods \\ Western blotting (WB)}

WB was performed to determine PD-1 and PD-L1 expressions in 4T1 mouse mammary tumor cell line (American Type Culture Collection, Manassas, VA, USA). Proteins were extracted in cold radioimmunoprecipitation assay lysis buffer, and protein concentrations of tumors were determined by bicinchoninic acid assay. Proteins were separated using 12\% acrylamide gel electrophoresis and transferred to polyvinylidene difluoride membranes. After 1-hour blocking in 10\% skim milk at room temperature, membranes were incubated with goat anti-mouse PD-1 monoclonal antibody (mAb) and rabbit anti-mouse PD-L1 polyclonal antibody (Thermo Scientific, Rockford, IL, USA) overnight at $4^{\circ} \mathrm{C}$. On the following day, the membranes were washed three times in Tris-buffered saline $(\mathrm{pH}=7.4,0.1 \%$ Tween-20) and incubated for 1 hour at room temperature in horseradish peroxidase-conjugated donkey anti-goat (Abcam, Burlingame, CA, USA) and anti-rabbit secondary IgG antibodies (R\&D Systems, Minneapolis, MN, USA). Blots were then developed by using an enhanced chemiluminescence system (Amersham Corp., Little Chalfont, UK).

\section{Immunohistochemistry (IHC)}

For IHC, tumor tissues were dissected and immediately fixed in $10 \%$ PBS buffer formalin overnight, then processed,

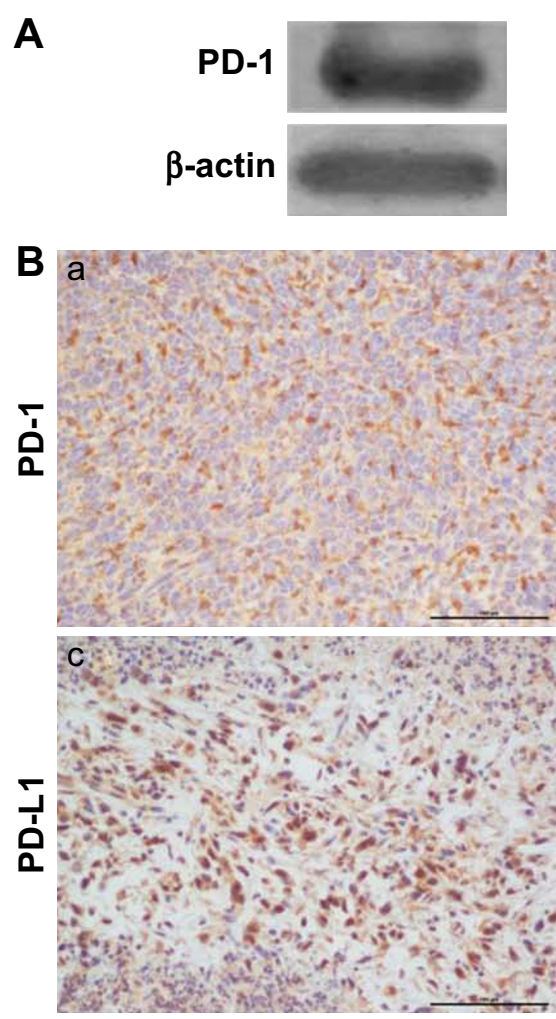

paraffin-embedded, sectioned, and stained according to standard protocols. The goat-anti-mouse PD-1 mAb and rabbit anti-mouse PD-L1 polyclonal antibody, or an equivalent concentration of IgG control, were used for staining.

\section{Analysis of tumor-infiltrated lymphocyte (TIL) composition}

The analysis of TIL composition was performed by fluorescence-activated cell sorting (FACS). Briefly, TILs were isolated from $4 \mathrm{~T} 1$ breast tumors after treatment with PD-1 $\mathrm{mAb}$ and stained with the indicated antibodies: antimouse CD45-eFluor 450, anti-mouse CD3-PerCP-eFluor 710, anti-mouse CD8-PE-Cyanine 7, anti-mouse CD25APC-eFluor 780, anti-mouse CD11b-APC (eBioscience, San Diego, CA, USA), anti-mouse PD1-PE, anti-mouse PD-L1-FITC (Tianjin Sungene Biotech Co., Ltd., Tianjin, China), isotype-matched control mIgG (Tianjin Sungene Biotech Co., Ltd.). FACS was performed on an FACSAria II (BD Biosciences, San Jose, CA, USA), and the data were analyzed by using Flow Jo 10.0.7 software (Tree Star, Ashland, OR, USA).
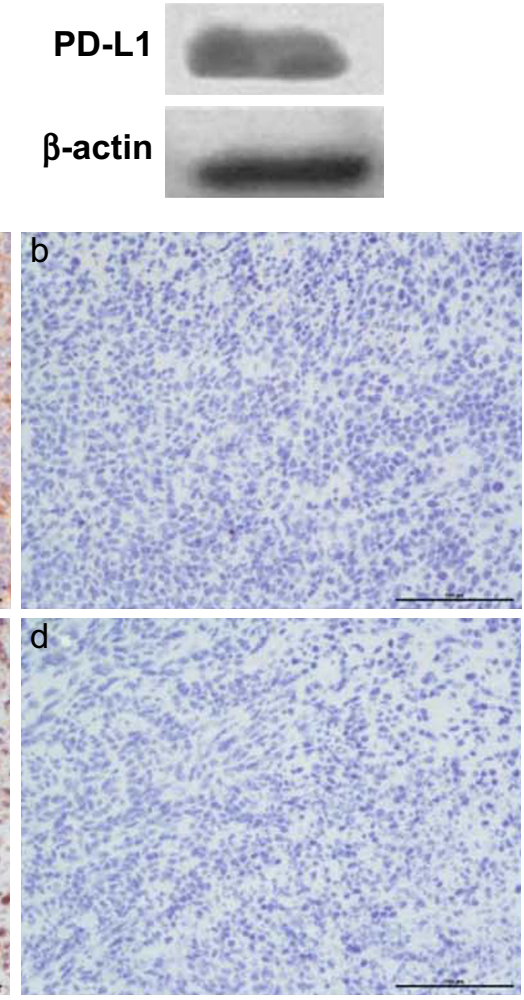

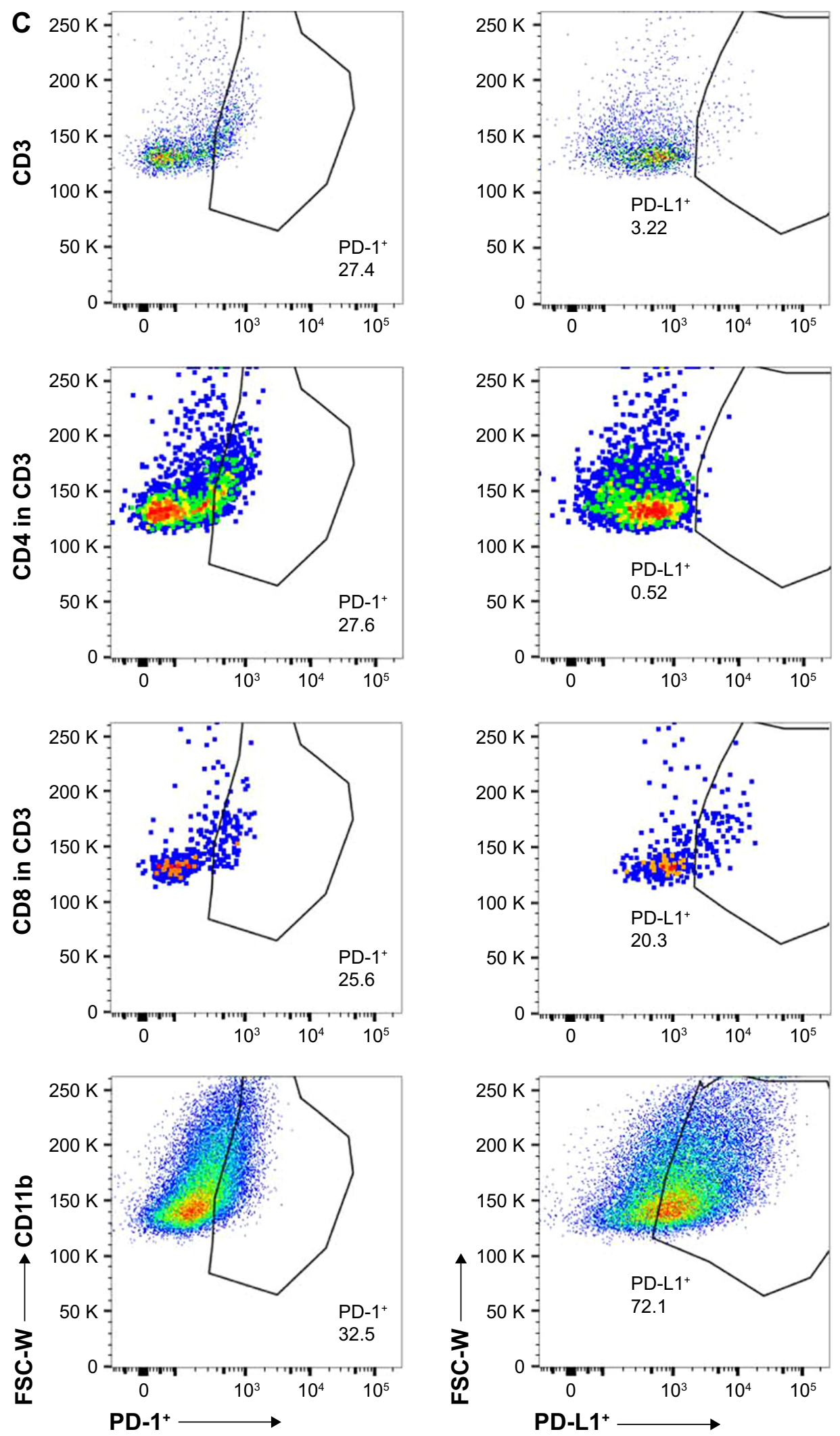

Figure SI PD-I and PD-LI expression analysis in 4TI mammary tumor tissues. (A) PD-I and PD-LI protein expressions were assessed in 4TI mammary tumor homogenates. (B) Immunohistochemical analysis of PD-I (a) and PD-LI expression (c) in 4TI mammary tumors. (b and d) lgG isotype staining served as a negative control for PD-I and PD-LI. Scale bar $=100 \mu \mathrm{m}$. (C) PD- $\mathrm{I}^{+}$and PD-LI ${ }^{+}$cell populations in TILs as determined by FACS $(n=3)$.

Abbreviations: FACS, fluorescence-activated cell sorting; TILs, tumor-infiltrated lymphocytes. 


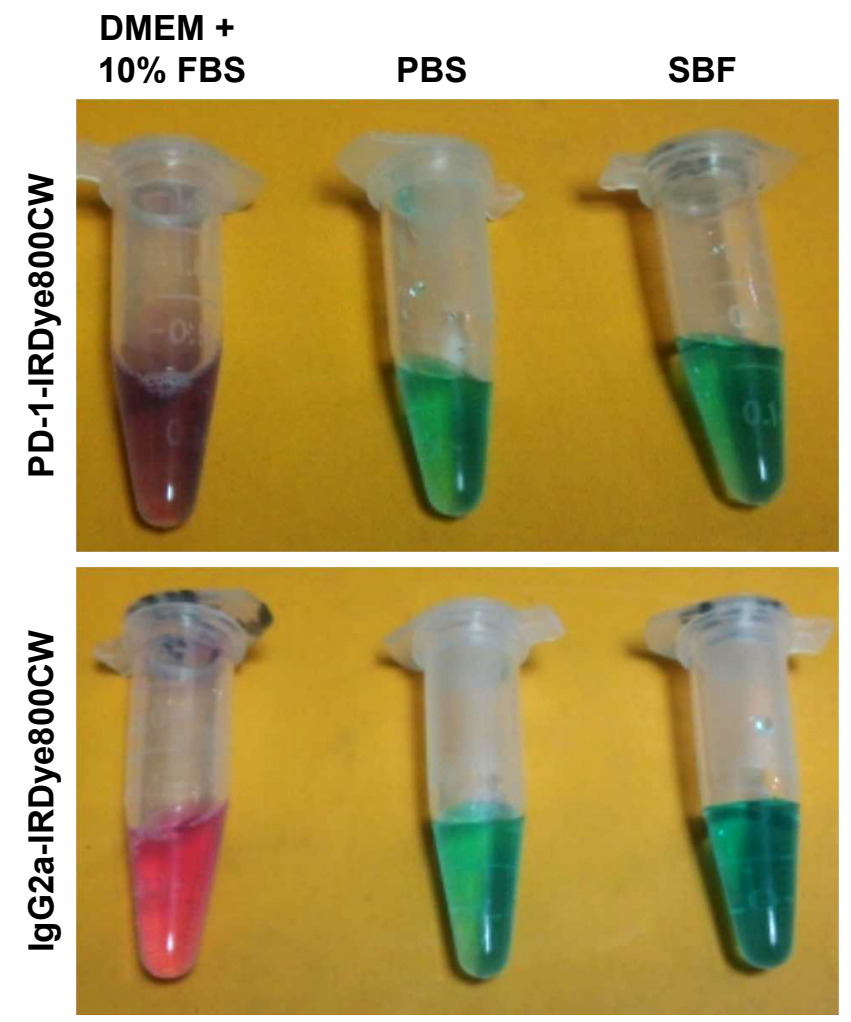

Figure S2 Photos of PD- I-IRDye800CW and IgG2a-IRDye800CW immunoconjugate dispersed in DMEM culture media containing I0\% FBS, PBS, and SBF. Abbreviations: FBS, fetal bovine serum; SBF, simulated body fluid; PBS, phosphate buffered saline.
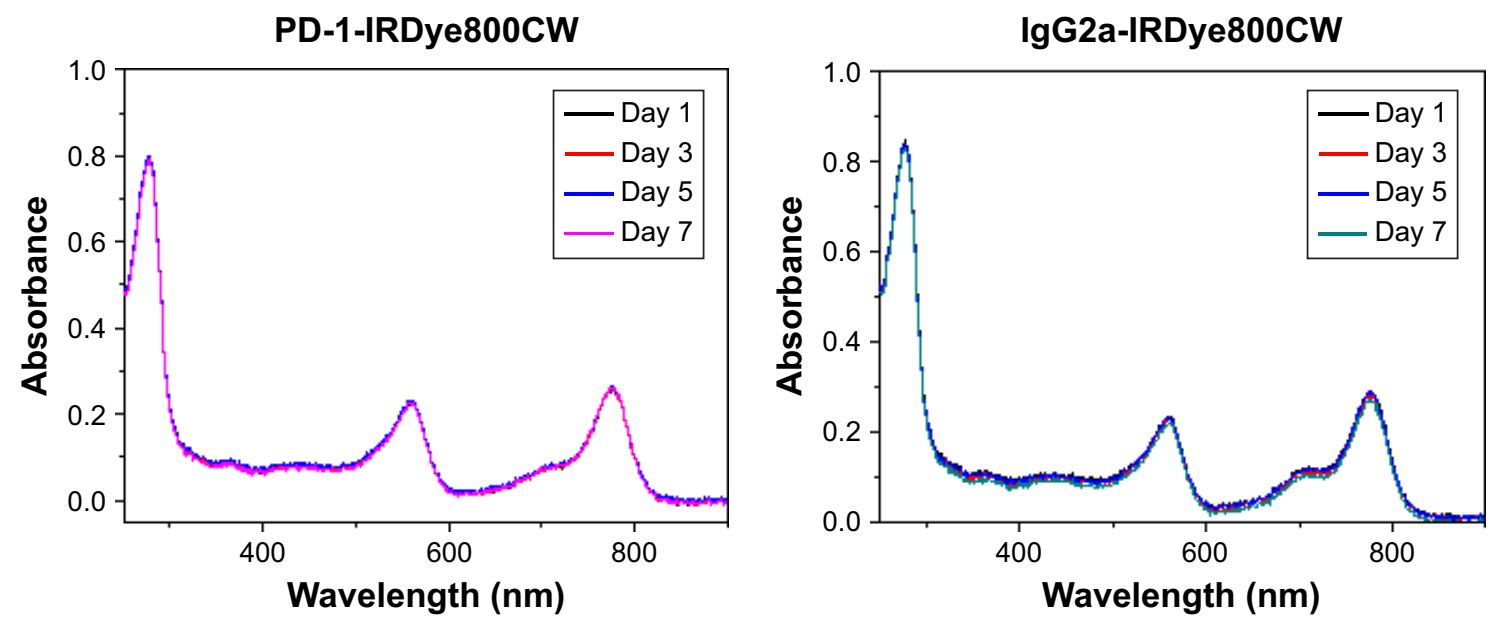

Figure S3 UV-Vis absorption spectra of PD-I-IRDye $800 \mathrm{CW}$ and IgG2a-IRDye $800 \mathrm{CW}$ immunoconjugate in DMEM culture media containing I0\% FBS changed overtime. The solution was stored at $4^{\circ} \mathrm{C}$ for 7 days.

Abbreviations: FBS, fetal bovine serum; UV-Vis, ultraviolet-visible. 

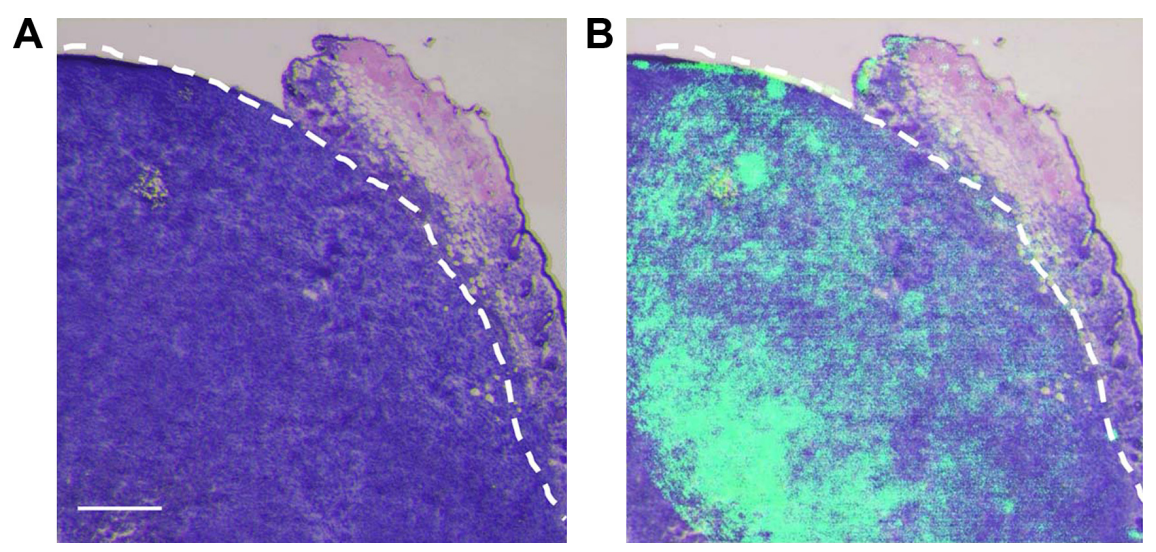

Figure S4 (A) Histological analysis of the tumor specimen encompassed the border regions between tumor and normal tissues from the resected tissues. (B) Overlay of PD-I NIRF image and H\&E staining of the specimen, showing consistent tumor margin at the microscopic level. Scale bar $=500 \mu \mathrm{m}$.

Abbreviation: NIRF, near-infrared fluorescence.

\section{Publish your work in this journal}

The International Journal of Nanomedicine is an international, peerreviewed journal focusing on the application of nanotechnology in diagnostics, therapeutics, and drug delivery systems throughou the biomedical field. This journal is indexed on PubMed Central, MedLine, CAS, SciSearch $®$, Current Contents $\AA /$ Clinical Medicine,
Journal Citation Reports/Science Edition, EMBase, Scopus and the Elsevier Bibliographic databases. The manuscript management system is completely online and includes a very quick and fair peer-review system, which is all easy to use. Visit http://www.dovepress.com/ testimonials.php to read real quotes from published authors.

\footnotetext{
Submit your manuscript here: http://www.dovepress.com/international-journal-of-nanomedicine-journal
} 